\title{
STONE DISEASE
}

\section{Computerized tomography guided access for percutaneous nephrostolithotomy} Matlaga BR, Shah OD, Zagoria RJ, Dyer RB, Streem SB, Assimos DG Department of Urology, Wake Forest University School of Medicine, Medical Center Boulevard, WinstonSalem, NC 27157, USA

J. Urol. 2003; 170: 45-7

Purpose: Access for percutaneous nephrostolithotomy (PNL) using conventional fluoroscopic guidance may carry an increased risk of damage to surrounding organs in patients with renal calculi and aberrant anatomy. In these situations cross-sectional anatomical imaging may facilitate safe percutaneous access. We describe our experience with computerized tomography (CT) guided percutaneous access for such patients undergoing PNL.

Materials and Methods: Between June 2000 and December 2001, 154 patients underwent PNL at our institution. Five of these patients (3\%) required a total of 6 percutaneous access tracks under CT guidance. All patients in this group had anatomical abnormalities precluding standard access to the collecting system without risk to adjacent organs. These abnormalities included a retrorenal colon in 2 and a severely distorted body habitus due to spinal dysraphism in 3.

Results: Percutaneous access was achieved without complication in all cases. At subsequent PNL 5 of the 6 renal units $(83 \%)$ were rendered completely stone-free.

Conclusion: CT guided percutaneous access is infrequently required for PNL. However, there is a select group of patients with anatomical anomalies that may predictably require this procedure to facilitate safe and efficacious PNL.

\section{Editorial Comment}

Aside from bleeding, the most common cause of morbidity associated with percutaneous nephrostolithotomy (PCNL) is injury to surrounding organs. With widespread use of CT imaging for the diagnosis of renal and ureteral calculi, anatomic features associated with risky percutaneous renal access are often identified. As the same time, patients with stones who are known to be at risk for anatomic anomalies often undergo CT imaging to evaluate the anatomic relations of the kidney to facilitate fluoroscopically-guided percutaneous access. For example, if CT shows that the spleen is located quite posteriorly and underlies the upper pole of the kidney in its lateral aspect, then the percutaneous puncture can be directed more medially under fluoroscopic guidance.

Matagla and colleagues, however, used CT guidance directly to obtain percutaneous renal access in patients at risk of injury with fluoroscopically-guided access. In doing so they reduced the chance of adjacent organ injury and increased the likelihood of satisfactory percutaneous renal access for PCNL. Although the risk of encountering a retrorenal colon, the most common cause (albeit rare) of colonic injury during PCNL, may not be sufficiently high to justify pre-operative CT imaging in all patient candidates for PCNL, those patients with known anatomic anomalies should undergo cross-sectional imaging as part of the routine preoperative planning process. For those few patients in whom percutaneous renal access cannot be safely obtained under fluoroscopic guidance, CT-guided access offers an effective means of achieving safe, optimal renal access.

Dr. Margaret S. Pearle

Associate Professor of Urology University of Texas Southwestern Med Ctr Dallas, Texas, USA 


\section{Variability of renal stone fragility in shock wave lithotripsy}

Williams JC Jr, Saw KC, Paterson RF, Hatt EK, McAteer JA, Lingeman JE

Department of Anatomy and Cell Biology, Indiana University School of Medicine, Indianapolis 46202, USA

Urology 2003; 61: 1092-7

Objectives: To measure, in an in vitro study, the number of shock waves to complete comminution for 195 human stones, representing six major stone types. Not all renal calculi are easily broken with shock wave lithotripsy. Different types of stones are thought to have characteristic fragilities, and suggestions have been made in published reports of variation in the fragility within some types of stones, but few quantitative data are available.

Methods: Kidney stones classified by their dominant mineral content were broken in an unmodified Dornier HM3 lithotripter or in a research lithotripter modeled after the HM3, and the number of shock waves was counted for each stone until all fragments passed through a sieve (3-mm-round or 2-mm-square holes).

Results: The mean +/- SD number of shock waves to complete comminution was $400+/-333$ per gram $(\mathrm{n}=39)$ for uric acid; $965+/-900$ per gram $(\mathrm{n}=75)$ for calcium oxalate monohydrate; $1134+/-770$ per gram $(\mathrm{n}=21)$ for hydroxyapatite; $1138+/-746$ per gram $(\mathrm{n}=13)$ for struvite; $1681+/-1363$ per gram $(\mathrm{n}=23)$ for brushite; and $5937+/-6190$ per gram $(\mathrm{n}=24)$ for cystine. The variation for these natural stones $(83 \%+/-15 \%$ coefficient of variation) was greater than that for artificial (eg, gypsum-based) stones $(17 \%+/-8 \%)$.

Conclusions: The variability in stone fragility to shock waves is large, even within groups defined by mineral composition. Thus, knowing the major composition of a stone may not allow adequate prediction of its fragility in lithotripsy treatment. The variation in stone structure could underlie the variation in stone fragility within type, but testing of this hypothesis remains to be done.

\section{Editorial Comment}

A number of clinical series have attempted to retrospectively correlate stone composition with success of shock wave lithotripsy (SWL). However, the ability to predict stone composition preoperatively on the basis of density on plain radiographs or attenuation on CT has been disappointing. Likewise, inconsistency in stone fragmentation among stones of similar composition has further limited our ability to predict SWL outcomes.

Williams and associates evaluated a series of human stones of different compositions as well as artificial stones to assess their susceptibility to and variability of fragmentation with SWL in vitro. Although uric acid and hydroxyapatite stones required the least and struvite and cystine stones the most shock waves to comminute, the variability within each group of stones with similar composition was remarkably high, suggesting that secondary factors, such as additional mineral components or variation in internal structure, also contribute to the overall susceptibility of a stone to SWL fragmentation.

This relatively simple but important study suggests that recent attempts to determine stone composition on the basis of radiographic characteristics may provide less predictive information than previously hoped. Although some generalizations may be made about susceptibility of stones of certain compositions to SWL fragmentation, in any individual case the outcome is less certain due to the large variability in response of stones to shock waves. Thus, knowledge of stone composition in and of itself may provide insufficient evidence on which to base patient selection for SWL.

Dr. Margaret S. Pearle Associate Professor of Urology University of Texas Southwestern Med Ctr Dallas, Texas, USA 